\title{
The ways of the scientific medical universe
}

$\mathrm{A}$

ny researcher wants to publish his paper, preferably in the most prestigious medical journal. This may confer competence, which is frequently merited. However, not publishing in the most prestigious journals does not indicate in competence, just as not all papers published in these journals are the most deserving.

In the first case, publication bias really exists. Thus publishing studies yielding negative results, even with excellent research, requires enormous persistence. Even discounting this bias, the most prestigious medical journals must normally reject 90 percent of the papers submitted to them. This means that many good papers are rejected for simple lack of space.

In the second case, editors are human and susceptible to many influences, so improperly-conducted research is sometimes published, thus increasing the "noise" in the medical literature and "obfuscating" sound evidence. To make the situation even worse, many universities customarily judge the individual scientific production of its researchers only by the number of publications, instead of on the quality and relevance of any publications. This "Publish or Perish" mentality simply encourages researchers to take the easy way out by producing "fast studies" of low quality, inspite of good evidence that took a long time to be gathered.
It is important that authors do not miss the point. It is our understanding that the goal is to properly conduct research in order to answer a relevant question. When completed, the results, whether positive or negative, need to be published so that they may belong to the universe of medical knowledge.

Nowadays, with the rapid and extensive development of information and communications systems, this task is becoming more simple. To open the door to the scientific universe, it is only necessary to publish a paper in a medical journal which is listed in the Index Medicus, Excerpta Medica, or Medline, as is the São Paulo Medical Journal. The knowledge contained in the paper will thus be easily accessable all over the planet.

Recently the São Paulo Medical Journal established an agreement with the editor of the Annals of Internal Medicine to evaluate the publication of good papers that have not obtained space in that journal, particularly the ones dealing with Evidence Based Medicine and Third World health problems. However, all authors with any properly-conducted study which answers relevant questions, short communications or case reports, are encouraged to try the São Paulo Medical Journal, in order to disseminate their knowledge throughout the universe of medical science. We have the space, and the good will. Good papers are welcome.

* MD, PhD, MSc

Chairman, Department of Internal Medicine, Escola Paulista de Medicina,

Editor, São Paulo Medical Journal. 